\title{
Surgical management of mesh-related complications after prior pelvic floor reconstructive surgery with mesh
}

\author{
Myrthe M. Tijdink • Mark E. Vierhout • \\ John P. Heesakkers • Mariëlla I. J. Withagen
}

Received: 29 January 2011 / Accepted: 30 May 2011 /Published online: 17 June 2011

(C) The Author(s) 2011. This article is published with open access at Springerlink.com

\begin{abstract}
Introduction and hypothesis The objective of this study is to evaluate the complications and anatomical and functional outcomes of the surgical treatment of mesh-related complications.

Methods A retrospective cohort study of patients who underwent complete or partial mesh excision to treat complications after prior mesh-augmented pelvic floor reconstructive surgery was conducted.

Results Seventy-three patients underwent 30 complete and 51 partial mesh excisions. Intraoperative complications occurred in 4 cases, postoperative complications in 13 . Symptom relief was achieved in $92 \%$ of patients. Recurrence of pelvic organ prolapse (POP) occurred in $29 \%$ of complete and $5 \%$ of partial excisions of mesh used in POP surgery. De novo stress urinary incontinence (SUI) occurred in $36 \%$ of patients who underwent excision of a suburethral sling.

Conclusions Mesh excision relieves mesh-related complications effectively, although with a substantial risk of serious complications and recurrence of POP or SUI. More complex excisions should be performed in skilled centers.
\end{abstract}

Keywords Complications · Excision · Exposure - Mesh · Pelvic organ prolapse $\cdot$ Stress urinary incontinence

M. M. Tijdink $(\bowtie) \cdot$ M. E. Vierhout $\cdot$ M. I. J. Withagen Department of Obstetrics and Gynaecology 791,

Radboud University Nijmegen Medical Centre,

P.O. Box 9101, $6500 \mathrm{HB}$, Nijmegen, the Netherlands

e-mail: myrthetijdink@hotmail.com

\section{J. P. Heesakkers}

Department of Urology,

Radboud University Nijmegen Medical Centre,

Nijmegen, the Netherlands

\author{
Abbreviations \\ IVS Intravaginal sling \\ OAB Overactive bladder \\ POP Pelvic organ prolapse \\ SUI Stress urinary incontinence \\ TOT Transobturator tape \\ TVT Tension-free vaginal tape \\ TVT-O Tension-free vaginal tape obturator
}

\section{Introduction}

Pelvic organ prolapse (POP) with or without stress urinary incontinence (SUI) is a major health issue in older women. The prevalence of POP-Q stage 2 or more varies from 37\% to $50 \%[1,2]$. A woman's lifetime risk of undergoing surgery for POP or SUI is approximately $11 \%$ [3]. Of those who undergo vaginal prolapse repair of the anterior vaginal wall, POP recurrence rates can reach 70\% [4]. Given this high recurrence rate after traditional vaginal prolapse surgery, many pelvic reconstructive surgeons began to use vaginal mesh to maintain the advantage of vaginal surgery and to obtain improved support. The use of synthetic mesh in procedures such as groin hernia surgery, abdominal sacrocolpopexy, and suburethral slings has been proven to be effective and is well established [5-7]. However, current data suggest that the use of mesh in vaginal prolapse surgery is associated with both benefits and risks. Few randomized controlled trials have been published. They reveal improved anatomic outcomes and lower recurrence rates, but similar subjective outcomes and substantial meshrelated complications after vaginal mesh placement when compared to traditional vaginal prolapse repairs [8-11].

Mesh-related complications include mainly mesh exposure [12] into the vagina or even the bladder or bowel, 
infection, granuloma, pain syndrome, dyspareunia, and voiding problems. Pelvic abscesses and rectovaginal or vesicovaginal fistula are also reported [13-17]. These complications occur in approximately $10 \%$ of patients and may lead to a significant decrease in quality of life [8-11, 18-20]. The extent of impact of mesh-related complications on quality of life has so far not been investigated thoroughly. Complications can be treated noninvasively in selected cases. However, with more severe mesh-related complications, partial or complete mesh excision is most of the time unavoidable. A recent review reported a higher rate of repeat surgery due to complications after vaginal mesh procedure compared to the rate of repeat surgery due to recurrent prolapse after conventional vaginal prolapse surgery [21].

The objective of this retrospective cohort study is to evaluate the surgical treatment of mesh-related complications with regard to complications and anatomical and functional outcomes.

\section{Materials and methods}

We conducted a retrospective cohort study including all patients who underwent surgical mesh excision, after prior POP and/or SUI surgery with mesh, between January 2005 and September 2010 in the Department of Obstetrics and Gynaecology of the Radboud University Nijmegen Medical Centre, a tertiary referral center in the Netherlands. In accordance with Dutch law, retrospective observational studies are exempt from submission for approval to a medical ethics review committee. Patients with meshrelated complications that were effectively managed on an outpatient basis were not included. Since we regarded voiding dysfunction after insertion of a suburethral sling a complication more specific for stress incontinence surgery than for vaginal mesh in general, we excluded these patients from further analysis (Fig. 1).

Patient characteristics, characteristics of mesh insertion procedure, relevant surgical history, POP-Q stage [22], SUI and overactive bladder $(\mathrm{OAB})$ complaints before mesh excision, symptoms and signs of mesh-related complications, and prior therapy were extracted from paper and electronic medical records. Operative reports and hospital discharge reports were used to collect characteristics of mesh excision, additional surgery, intraoperative complications, postoperative complications, and hospital stay. Outcomes after mesh excision including relief of mesh-related symptoms, mesh exposure, recurrence of POP, de novo SUI and $\mathrm{OAB}$, and need of re-excision were collected at the latest available moment of follow-up. The extent of symptom relief was determined by patient history and categorized into complete relief when patients expressed no remaining symptoms, improvement when some symptoms persisted, and no improvement when symptoms had been unchanged. We defined recurrence of POP as the development of at least POP stage 2 after mesh excision or increase of POP with at least one stage when there was POP stage 2 before in the compartment where mesh was excised or occurring after apical mesh excision.

Patients who underwent partial mesh excision, including section of mesh, were compared to patients who underwent complete mesh excision. Continuous variables were compared using the Mann-Whitney $U$ test. Categorical variables were compared using the chi-square test and, in case of small numbers, with Fisher's exact test. A $P$ value of $\leq 0.05$
Fig. 1 Flowchart of inclusion of patients who underwent excision of mesh

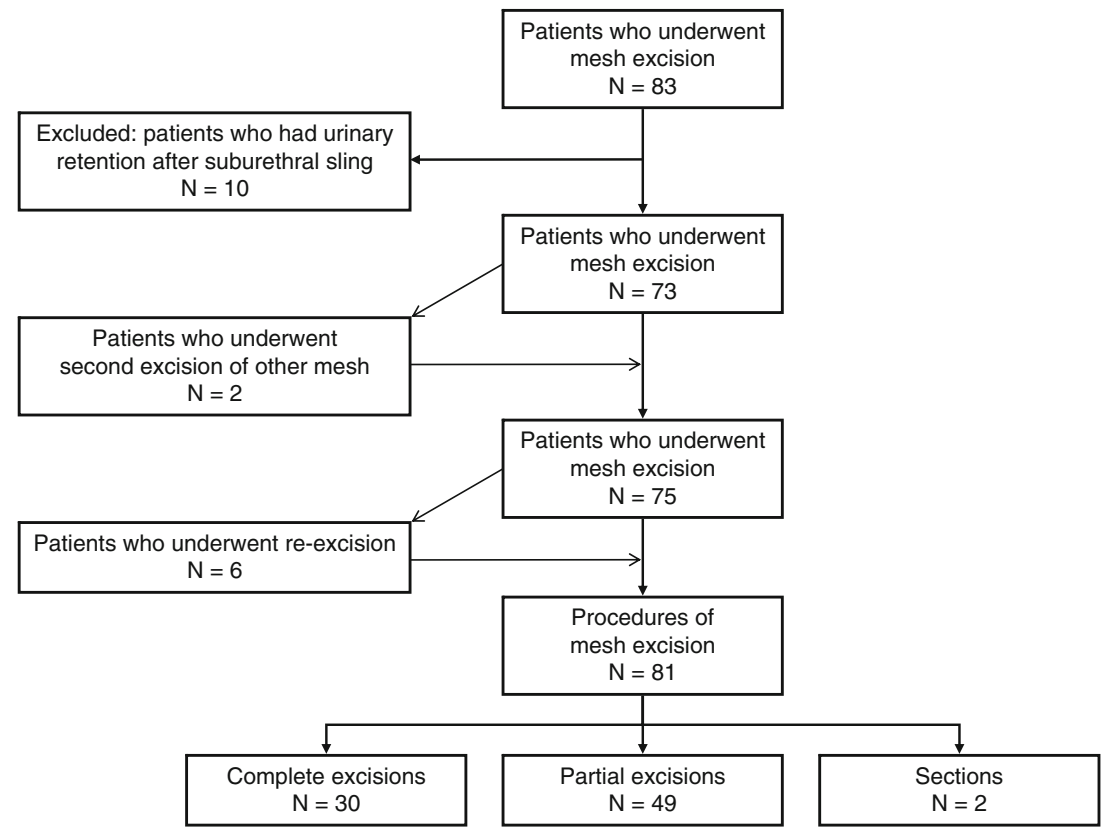


was considered statistically significant. Data were analyzed using SPSS version 16.0 (SPSS Inc., Chicago, IL, USA).

\section{Surgical technique}

An examination under anesthesia was performed and the problematic area of the mesh was identified. When there was suspicion of mesh exposure in the bladder or bowel, cystoscopy and/or rectal examination was used to identify the presence of exposed mesh in the bladder or rectum.

\section{Partial vaginal mesh excision}

In case of a limited vaginal exposure with mild symptoms, the exposure site was circumcised and the edges of the vaginal epithelium were mobilized approximately $1 \mathrm{~cm}$ around the exposure. The extruded part of the mesh was then removed and the edges of the vaginal epithelium trimmed, where appropriate, and reapproximated.

\section{Complete mesh excision via the vaginal approach}

When there were larger exposures, severe symptoms, and/ or involvement of the bladder or bowel (described in the "Results" section), the intention was to perform total mesh excision or to remove as much mesh as possible in case of mesh arms passing through the obturator space, ischiorectal fossa, or sacrum. The vaginal epithelium covering the mesh was injected with normal saline or $0.5 \%$ lidocaine with 1:200,000 epinephrine for hydrodissection. A midline or inverted U-shaped full-thickness incision was performed over the palpable mesh and vaginal epithelium was sharply dissected from the mesh as far laterally as possible. The mesh, where necessary with a layer of interlaced fibrotic tissue, was carefully dissected from the bladder or rectum using both sharp and gentle blunt dissection. Sharp dissection of posterior mesh was performed over a finger placed into the rectum to avoid rectal injury. Once the body of the mesh was freed and the mesh arms were visualized, tension was applied to expose the maximum amount of mesh. The body of the mesh was then separated from the mesh arms at the most lateral position possible. In some cases of excision of sacrocolpopexy mesh, a hysteroscope was used to enable a higher and thus more complete mesh removal. When hemostasis was achieved, the vaginal epithelium was reapproximated. If necessary, concomitant procedures for prolapse repair or other gynecologic disorders were performed.

When the tape was also exposed intravesically, firstly, two ureteral stents were inserted. The bladder was opened through the pubocervical fascia and the tape excised, taking utmost care of the ureters. The bladder was then closed in two layers, taking care to avoid the ureters. The ureteral stents were removed at the end of the procedure. The bladder was drained for 7-14 days with an indwelling catheter. An ultrasound control of the kidneys was performed after 1 day.

\section{Abdominal mesh excision}

An abdominal approach was applied for the excision of mesh used in sacrocolpopexy or suburethral slings when previous vaginal approach has not been successful or in case of extensive bladder involvement.

In abdominal excision of sacrocolpopexy mesh, the abdominal cavity was accessed through a vertical midline incision. The intestines were packed out of the pelvis with moist laparotomy gauzes and held in place with a retractor. Adhesiolysis was performed as necessary to allow adequate visualization. The mesh was removed from the sacrum and apex of the vagina by both sharp and blunt dissection. The vaginal vault was closed, leaving a small opening for postoperative drainage. For abdominal excision of transvaginal tape (TVT) or excision of other intravesical localized mesh, a Pfannenstiel incision allowed entry to the Retzius space. After localizing the tape, it was dissected from the abdominal wall and bladder.

In case of an intravesical exposure, dissection was continued down to the point of entry of the tape into the bladder, the bladder was opened, and the mesh was removed completely. The bladder was then closed in two layers, taking care to avoid the ureters. If necessary, any remaining mesh in the vagina was removed by a vaginal approach as described above. The bladder was drained for 7-14 days with an indwelling catheter. When the resection had to be done in proximity of the ureters, an ultrasound control of the kidneys was performed after the mesh excision procedure and after the removal of the ureteral stents.

\section{Results}

Between January 2005 and September 2010, 83 patients underwent surgical excision of mesh used in POP or SUI surgery. Ten patients required excision of mesh because of urinary retention after insertion of a suburethral sling and were withdrawn from further analysis. Of the remaining 73 patients who underwent mesh excision, 2 patients underwent a second excision for other mesh in another compartment. In further analysis, these 2 patients were considered as 4 individuals, resulting in 75 patients. Six patients required a re-excision of the same mesh. Overall, there were 81 operations for mesh excision, of which 30 (37\%) were complete excisions, 49 (61\%) partial excisions, and $2(2 \%)$ sections of mesh (Fig. 1). In further analysis, sections of mesh were considered as partial mesh excisions. 
Patient demographics and characteristics before mesh excision are summarized in Table 1. A variety of mesh materials were excised in our population: Gynemesh ${ }^{\mathrm{TM}}$,

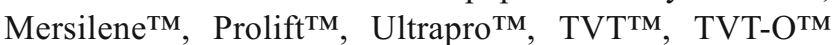
(Ethicon, Somerville, NJ, USA), IVS ${ }^{\mathrm{TM}}$ (Tyco Healthcare LP, Norwalk, CT, USA), Avaulta ${ }^{\mathrm{TM}}$ (CR Bard, Covington, GA, USA), Apogee ${ }^{\mathrm{TM}}$ (American Medical Systems, Minnetonka, MN, USA), EndoFast Reliant ${ }^{\mathrm{TM}}$ (Endogun Medical Systems, Kibbutz Haogen, Israel), Gore-Tex ${ }^{\mathrm{TM}}$, Mycromesh ${ }^{\mathrm{TM}}$ (WL Gore, Flagstaff, AZ, USA), Teflon ${ }^{\mathrm{TM}}$ (Davol/Bard, Cranston, RI, USA). Prolift ${ }^{\mathrm{TM}}$ was the most removed mesh material in our patients $(40 \%)$. The mesh insertion surgery was performed at our center in $41 \%$ of patients; in $59 \%$, mesh was inserted at other hospitals.

Frequency of signs and symptoms and the distribution between different types of mesh insertion procedure are presented in Table 2. Fifty-seven (76\%) patients had mesh exposure, of which most were localized in the vaginal wall $(90 \%)$. Furthermore, four patients had an exposure in the bladder (three after TVT ${ }^{\mathrm{TM}}$, one after Prolift ${ }^{\mathrm{TM}}$ ), two patients had a vesicovaginal fistula (after Prolift ${ }^{\mathrm{TM}}$ ), and one patient had a rectovaginal fistula (after sacrocolpopexy). Most women reported more than one mesh-related symptom. Only one patient did not report any symptoms, although she had a persistent, large exposure of the mesh. The primary complaint was pain in the majority of cases (77\%), which consisted of dyspareunia, vaginal pain, chronic pain of the abdomen, back, buttock, or leg, or a combination of these. A distinct difference in frequency of mesh-related symptoms existed between the different types of mesh insertion procedure, especially in sacrocolpopexy compared to the other procedures (Table 2). Pain and dyspareunia are mainly seen after vaginal mesh insertion and vaginal bleeding and discharge after sacrocolpopexy. Patients with mesh exposure also presented differently than patients without exposure. They reported less pain symptoms (54\% vs 94\%, $P=0.002)$ and more often vaginal bleeding and discharge (32\% vs $6 \%, P=0.031$ and $47 \%$ vs $6 \%, P=0.002$ ).

Patients with severe mesh complications, patients with exposure in the bladder or fistula, and patients that have had a previous mesh excision underwent more often

Table 1 Patient demographics and preoperative characteristics

\begin{tabular}{|c|c|c|c|c|}
\hline & $\begin{array}{l}\mathrm{All}, N=75 \\
(100 \%)\end{array}$ & $\begin{array}{l}\text { Partial excision, } n=48 \\
(64 \%)\end{array}$ & $\begin{array}{l}\text { Complete excision, } n=27 \\
(36 \%)\end{array}$ & $P$ value \\
\hline Age (years) & $56 \pm 11$ & $54 \pm 10$ & $59 \pm 10$ & 0.034 \\
\hline $\mathrm{BMI}^{\mathrm{a}}$ & $26 \pm 4$ & $26 \pm 4$ & $26 \pm 3$ & 0.822 \\
\hline Smoking ${ }^{\mathrm{b}}$ & $12(16)$ & $11(23)$ & $1(4)$ & 0.046 \\
\hline Comorbidity & $49(65)$ & $33(69)$ & $16(59)$ & 0.407 \\
\hline History of POP/SUI surgery prior to mesh insertion & $56(75)$ & $35(73)$ & $21(78)$ & 0.642 \\
\hline No. of previous POP/SUI surgeries & $2(0-7)$ & $2(0-7)$ & $2(0-4)$ & 0.835 \\
\hline Previous hysterectomy & $58(77)$ & $35(73)$ & $23(85)$ & 0.223 \\
\hline \multicolumn{5}{|l|}{ Procedure mesh insertion } \\
\hline Anterior repair & $14(19)$ & $7(15)$ & $7(26)$ & 0.226 \\
\hline Posterior repair & $15(20)$ & $13(27)$ & $2(7)$ & 0.041 \\
\hline Total mesh/anterior-posterior repair & $19(25)$ & $14(29)$ & $5(19)$ & 0.309 \\
\hline Sacrocolpopexy & $12(16)$ & $5(10)$ & $7(26)$ & 0.104 \\
\hline Retro pubic incontinence sling & $6(8)$ & $3(6)$ & $3(11)$ & 0.661 \\
\hline Transobturator incontinence sling & $9(12)$ & $6(13)$ & $3(11)$ & 1.000 \\
\hline \multicolumn{5}{|l|}{ POP-Q stage before mesh excision ${ }^{c}$} \\
\hline POP-Q stage 0 & $31(43)$ & $18(39)$ & $13(50)$ & 0.371 \\
\hline POP-Q stage I & $11(15)$ & $10(22)$ & $1(4)$ & 0.048 \\
\hline POP-Q stage II & $25(35)$ & $16(35)$ & $9(35)$ & 0.989 \\
\hline POP-Q stage III & $5(7)$ & $2(4)$ & $3(12)$ & 0.344 \\
\hline Former mesh excision & $22(29)$ & $6(13)$ & $16(59)$ & $<0.001$ \\
\hline No. of former mesh excisions & $0(0-4)$ & $0(0-1)$ & $1(0-4)$ & $<0.001$ \\
\hline
\end{tabular}

Values are presented as mean $\pm \mathrm{SD}$, median (range), or number (\%). $P$ value represents a comparison between the groups "partial excision" and "complete excision"

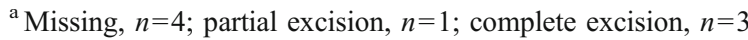

${ }^{\mathrm{b}}$ Missing, $n=1$; partial excision, $n=1$

${ }^{\mathrm{c}}$ Missing, $n=3$; partial excision, $n=2$; complete excision, $n=1$ 
Table 2 Presenting mesh-related symptoms and signs per type of mesh insertion procedure

\begin{tabular}{|c|c|c|c|c|c|}
\hline & $\begin{array}{l}\text { All, } N=75 \\
(100 \%)\end{array}$ & $\begin{array}{l}\text { Sacrocolpopexy, } \\
n=12(16 \%)\end{array}$ & $\begin{array}{l}\text { Vaginal POP repair, } \\
n=48(64 \%)\end{array}$ & $\begin{array}{l}\text { Suburethral slings, } \\
n=15(20 \%)\end{array}$ & $P$ value \\
\hline Exposure & $57(76)$ & $11(92)$ & $34(71)$ & $12(80)$ & 0.294 \\
\hline Vagina & $51(90)$ & $10(91)$ & $31(91)$ & $10(83)$ & 0.737 \\
\hline Bladder & $3(5)$ & $0(0)$ & $1(3)$ & $2(17)$ & 0.128 \\
\hline Vesicovaginal fistula & $2(4)$ & $0(0)$ & $2(6)$ & $0(0)$ & 0.496 \\
\hline Rectovaginal fistula & $1(2)$ & $1(9)$ & $0(0)$ & $0(0)$ & 0.119 \\
\hline \multicolumn{6}{|l|}{ Symptoms } \\
\hline Vaginal bleeding & $19(25)$ & $9(75)$ & $8(17)$ & $2(13)$ & $<0.001$ \\
\hline Vaginal discharge & $28(37)$ & $10(83)$ & $16(33)$ & $2(13)$ & 0.001 \\
\hline Dyspareunia & $42(56)$ & $2(17)$ & $31(65)$ & $9(60)$ & 0.011 \\
\hline Pain (vaginal pain and/or chronic pain) & $48(64)$ & $3(25)$ & $34(71)$ & $11(73)$ & 0.009 \\
\hline Vaginal pain & $43(57)$ & $2(17)$ & $32(67)$ & $9(60)$ & 0.007 \\
\hline Chronic pain & $32(43)$ & $2(17)$ & $23(48)$ & $7(47)$ & 0.138 \\
\hline Abdomen & $17(53)$ & $1(50)$ & $12(52)$ & $4(57)$ & 0.970 \\
\hline Back & $3(9)$ & $0(0)$ & $3(13)$ & $0(0)$ & 0.523 \\
\hline Buttock & $8(25)$ & $1(50)$ & $7(30)$ & $0(0)$ & 0.186 \\
\hline Leg & $4(13)$ & $0(0)$ & $1(4)$ & $3(43)$ & 0.023 \\
\hline Urinary tract infections & $4(5)$ & $3(25)$ & $0(0)$ & $1(7)$ & 0.003 \\
\hline Defecation problems & $8(11)$ & $2(17)$ & $6(13)$ & $0(0)$ & 0.299 \\
\hline Dysfunctional voiding & $3(4)$ & $1(8)$ & $1(2)$ & $1(7)$ & 0.516 \\
\hline Severe mesh complications & $15(20)$ & $1(8)$ & $13(27)$ & $1(7)$ & 0.123 \\
\hline Mesh shrinkage & $8(11)$ & $0(0)$ & $8(17)$ & $0(0)$ & 0.081 \\
\hline Mesh displacement & $1(1)$ & $0(0)$ & $1(2)$ & $0(0)$ & 0.752 \\
\hline Chronic inflammation & $2(3)$ & $0(0)$ & $2(4)$ & $0(0)$ & 0.561 \\
\hline Infection & $1(1)$ & $0(0)$ & $1(2)$ & $0(0)$ & 0.752 \\
\hline Granuloma & $7(9)$ & $1(8)$ & $5(10)$ & $1(7)$ & 0.902 \\
\hline
\end{tabular}

Values are presented as number (\%). $P$ value represents a comparison between the groups "sacrocolpopexy," "vaginal POP repair," and "suburethral slings"

complete mesh excision compared to patients who did not have these severe complications $(73 \%$ vs $27 \%, P=0.001$, $83 \%$ vs $29 \%, P=0.017$, and $73 \%$ vs $21 \%, P<0.001$, respectively).

Mesh-related complications were unsuccessfully treated conservatively with estrogen cream, antibiotics, and/or physiotherapy prior to mesh excision in $63 \%$ of patients. Previous mesh excision procedures were performed in 22 patients $(29 \%)$. Five patients had two or more previous excisions. Three patients had undergone an earlier attempted complete excision.

Characteristics of mesh excision surgery are presented in Table 3. Complete mesh excisions were frequently assisted by a urologist, especially in complex cases and with bladder involvement. Mesh was most often (89\%) excised by a vaginal approach. In five cases, abdominal approach was combined with vaginal excision, and in one case, it was a laparoscopic approach. Additional POP repair surgery was performed in 14 patients $(17 \%)$. These were, except for one patient, classical repairs without mesh and in 10 out of 14 of another compartment then where the mesh was removed. Additional surgery led to a longer operation time (mean, $66 \pm$ 24 vs $35 \pm 31$ min; $P<0.001$ ).

Intraoperative complications occurred during four surgical procedures: three patients had a bowel lesion (one perforation of the sigmoid and two serosal lesions of the colon and small bowel), which occurred during abdominal mesh excision for previous sacrocolpopexy and were repaired during surgery. Postoperatively, one patient developed anuria as a consequence of lesions to both ureters, which had remained unnoticed during surgery. During this procedure of complete vaginal excision of mesh located at the anterior vaginal wall, dissection of the mesh from the bladder wall was difficult. However, at that moment, there was no suspicion of damage to the ureters. A bladder lesion was excluded by filling the bladder with methylene blue dye. Once this serious complication was identified, she was treated successfully with bilateral ureter reimplantation. 
Table 3 Characteristics of mesh excision surgery

\begin{tabular}{|c|c|c|c|c|}
\hline & $\begin{array}{l}\text { All, } N=81 \\
(100 \%)\end{array}$ & $\begin{array}{l}\text { Partial excision, } \\
n=51(63 \%)\end{array}$ & $\begin{array}{l}\text { Complete excision, } \\
n=30(37 \%)\end{array}$ & $P$ value \\
\hline Time between mesh insertion and recent excision (months) & $16(2-217)$ & $11(2-208)$ & $26(5-217)$ & 0.001 \\
\hline Assistance of urologist & $10(12)$ & $1(2)$ & $9(30)$ & $<0.001$ \\
\hline Operation time (min) & $43 \pm 29$ & $34 \pm 26$ & $58 \pm 28$ & $<0.001$ \\
\hline Blood loss (ml) & $77 \pm 147$ & $52 \pm 107$ & $120 \pm 193$ & 0.066 \\
\hline \multicolumn{5}{|l|}{ Site of mesh excision } \\
\hline Anterior vaginal wall & $22(27)$ & $14(27)$ & $8(27)$ & 0.939 \\
\hline Posterior vaginal wall & $18(22)$ & $14(27)$ & $4(13)$ & 0.140 \\
\hline Anterior and posterior vaginal wall & $5(6)$ & $5(10)$ & $0(0)$ & 0.152 \\
\hline Anterior and posterior vaginal wall and vaginal vault & $2(2)$ & $0(0)$ & $2(7)$ & 0.134 \\
\hline Vaginal vault & $22(27)$ & $10(20)$ & $12(40)$ & 0.046 \\
\hline Suburethral & $19(23)$ & $11(21)$ & $8(27)$ & 0.601 \\
\hline \multicolumn{5}{|l|}{ Approach } \\
\hline Vaginal $^{\mathrm{a}}$ & $72(89)$ & $49(96)$ & $23(77)$ & 0.011 \\
\hline Abdominal $^{\mathrm{b}}$ & $9(11)$ & $2(4)$ & $7(23)$ & 0.011 \\
\hline Use of hysteroscope & $2(2)$ & $0(0)$ & $2(7)$ & 0.134 \\
\hline Use of laparoscope & $1(1)$ & $1(2)$ & $0(0)$ & 1.000 \\
\hline Additional surgery & $21(26)$ & $13(25)$ & $8(27)$ & 0.907 \\
\hline POP & $14(17)$ & $8(16)$ & $6(20)$ & 0.762 \\
\hline SUI & $3(4)$ & $2(4)$ & $1(3)$ & 1.000 \\
\hline Other & $4(5)$ & $3(6)$ & $1(3)$ & 0.609 \\
\hline Intraoperative complications & $4(5)$ & $1(2)$ & $3(10)$ & 0.141 \\
\hline Postoperative complications & $13(16)$ & $5(10)$ & $8(27)$ & 0.062 \\
\hline Hospital stay (days) & $2 \pm 2$ & $1 \pm 1$ & $3 \pm 2$ & $<0.001$ \\
\hline
\end{tabular}

Values are presented as mean $\pm \mathrm{SD}$, median (range), or number (\%). $P$ value represents a comparison between the groups "partial excision" and "complete excision"

${ }^{a}$ Two combined with hysteroscopy

${ }^{\mathrm{b}}$ Five combined with vaginal excision, one with laparoscope

Postoperative complications occurred after 13 surgical procedures, including hematoma $(n=2)$, substantial bleeding $(n=1)$, ileus $(n=1)$, urinary tract infection $(n=5)$, urinary retention $(n=3)$, fever $(n=2)$, wound infection $(n=$ $1)$, subcutaneous abscess $(n=1)$, fistula from cervix to sigmoid $(n=1)$, obstruction of a unilateral ureter and consequently blow-out of the kidney $(n=1)$. The latter serious complication occurred in a patient who appeared to have a bilateral relative ureteropelvic junction stenosis, which probably was aggravated by the surgery. She was treated by temporary insertion of a nephrostomy catheter. Complications seemed to be more frequent in the group with complete mesh excision, although this difference was not statistically significant.

Complications were more common in the group with former sacrocolpopexy; intraoperative complications in this group occurred in $23 \%$ (vs $1 \%, P=0.001$ ) and, only during abdominal excision, postoperative complications in this group occurred in $46 \%$ (vs $12 \%, P=0.003$ ). All patients recovered completely after treatment of their complications.
Outcomes are shown in Table 4. The majority of patients had relief, either complete relief or improvement, of meshrelated symptoms (92\%). No difference was found between groups with regard to symptom relief, although five of six patients with no relief of symptoms had undergone a partial excision. Nine patients (12\%) had recurrence of POP, of which eight were in the anterior compartment. The recurrence rate of POP was significantly higher in patients who underwent complete excision of mesh used in POP surgery $(29 \%)$ compared to patients who underwent partial excision (5\%). De novo SUI after mesh excision was more frequent in the group of patients who underwent excision of suburethral slings (36\%) compared to patients who underwent excision of mesh used in POP surgery $(7 \%, P=0.010)$.

Six patients $(8 \%)$ needed re-excision of mesh; all had undergone a partial excision before. Four had persistent exposure of mesh in the vagina, one had a new exposure of mesh in the bladder, while she earlier had an exposure of mesh in the vagina, and one had persistent pain. Three patients underwent additional complete excision and three 
Table 4 Outcomes of mesh excision

All, $N=75(100 \%) \quad$ Partial excision, $n=48(64 \%) \quad$ Complete excision, $n=27(36 \%) \quad P$ value

\begin{tabular}{|c|c|c|c|c|}
\hline Relief of mesh-related symptoms & $69(92)$ & $43(90)$ & $26(96)$ & 0.410 \\
\hline No improvement & $6(8)$ & $5(10)$ & $1(4)$ & 0.410 \\
\hline Improvement & $21(28)$ & $14(29)$ & $7(26)$ & 0.764 \\
\hline Complete relief & $48(64)$ & $29(60)$ & $19(70)$ & 0.389 \\
\hline Persistent exposure after mesh excision & $6(8)$ & $6(13)$ & $0(0)$ & 0.082 \\
\hline \multicolumn{5}{|l|}{ POP-Q stage after mesh excision ${ }^{a}$} \\
\hline POP-Q stage 0 & $24(35)$ & $14(32)$ & $10(42)$ & 0.417 \\
\hline POP-Q stage I & $18(27)$ & $15(34)$ & $3(13)$ & 0.054 \\
\hline POP-Q stage II & $19(28)$ & $14(32)$ & $5(21)$ & 0.335 \\
\hline POP-Q stage III & $7(10)$ & $1(2)$ & $6(25)$ & 0.006 \\
\hline Recurrence of $\mathrm{POP}^{\mathrm{b}}$ & $9(12)$ & $3(6)$ & $6(23)$ & 0.061 \\
\hline Recurrence of POP (POP mesh only, $n=60)^{\mathrm{c}}$ & $8(14)$ & $2(5)$ & $6(29)$ & 0.019 \\
\hline De novo SUI after mesh excision ${ }^{\mathrm{d}}$ & $9(12)$ & $5(11)$ & $4(15)$ & 0.712 \\
\hline De novo OAB after mesh excision ${ }^{\mathrm{e}}$ & $6(8)$ & $2(4)$ & $4(15)$ & 0.178 \\
\hline Follow-up (months) & $6(0-50)$ & $5(0-50)$ & $7(1-42)$ & 0.557 \\
\hline Need of re-excision & $6(8)$ & $6(13)$ & $0(0)$ & 0.082 \\
\hline
\end{tabular}

Values are presented as median (range) or number (\%). $P$ value represents a comparison between the groups "partial excision" and "complete excision"

${ }^{a}$ Missing, $n=7$; partial excision, $n=4$; complete excision, $n=3$

${ }^{\mathrm{b}}$ Missing, $n=2$; partial excision, $n=1$; complete excision, $n=1$

${ }^{\mathrm{c}}$ Missing, $n=1$; partial excision, $n=1$

${ }^{\mathrm{d}}$ Missing, $n=2$; partial excision, $n=1$; complete excision, $n=1$

${ }^{\mathrm{e}}$ Missing, $n=2$; partial excision, $n=1$; complete excision, $n=1$

had partial excision. Eventually, after this re-excision, five patients had complete relief and one had improvement of symptoms, none had exposure, none had recurrence of POP, one had de novo SUI, and one had de novo OAB.

The group of patients who underwent excision of mesh used in sacrocolpopexy was not different from the other groups concerning the outcomes. All had relief of symptoms $(23 \%$ improvement and $77 \%$ complete relief of symptoms), none had exposure, three (25\%) had recurrence of POP, one had SUI and one had OAB after mesh excision, and one patient needed re-excision of mesh.

\section{Discussion}

In this retrospective cohort study, we demonstrated that surgical mesh excision to treat complications after prior POP or SUI surgery with mesh was successful in the majority of cases, although with a substantial risk of serious complications and recurrence of POP or SUI. Only $8 \%$ of patients experienced no improvement of their symptoms. Main indications for mesh removal included pain (dyspareunia, vaginal pain, and/or pain in the lower abdomen, back, buttock, or leg), vaginal bleeding, and discharge, with or without evident mesh exposure. The frequency of these symptoms differed substantially between different types of mesh insertion procedure, but was similar to numbers found in other studies [14-17, 23, 24]. Pain and dyspareunia are mainly seen after vaginal mesh insertion and vaginal discharge and bleeding after sacrocolpopexy.

Mesh exposures are often reported as painless $[8,9,16]$; however, pain is still found frequently together with exposures [14, 15, 17]. In our study, pain and mesh exposure were both frequently reported; however, patients with mesh exposure reported less pain symptoms than patients without exposure. Still, 54\% of patients with mesh exposure did report pain symptoms (excluding dyspareunia, which is often already present before mesh insertion [8, 20]). The exposure itself may not have been the cause of pain. Underlying mesh contraction, although less severe contraction may not always be recognized at physical examination, could actually have induced the pain symptoms [25]. It may also be possible that more complex cases of mesh exposures were seen at our tertiary referral center, in which pain may be more frequently present than in patients with less severe exposures.

Most of the recent publications on vaginal mesh surgery report only short-term and medium-term follow-up results. Indeed, most of the mesh complications occur within the first postoperative year [9]. However, late complications 
may arise after a long period of time. We found meshrelated complications even up to 18 years after mesh insertion and, in $36 \%$ of mesh excisions, the time between mesh insertion and removal was more than 2 years, mostly after sacrocolpopexy. Marcus-Braun et al. found meshrelated complications up to 8 years after mesh placement; in $56 \%$, the delay from the primary operation was more than 2 years [13]. Time between mesh insertion and excision was longer in the group of patients who underwent complete mesh excision, probably because they have undergone more minor interventions (former partial mesh excision) to treat their complications in this period.

Not every mesh-related complication requires complete excision, although complete excision of mesh is sometimes advocated to avoid multiple procedures and to achieve optimal relief of symptoms [14, 24, 26]. In our study, most patients $(64 \%)$ underwent partial excision of mesh. Nevertheless, we found no difference in relief of symptoms between partial and complete excision. Ridgeway et al. described 19 cases of mesh removal in which the degree of mesh excision was tailored to the severity of the complications. This resulted also in great alleviation of symptoms in most cases [16]. In our series, patients with more serious complaints indeed underwent more often a complete mesh excision.

A substantial number of patients $(36 \%)$ required multiple procedures, of which most had been performed in other centers before referral to our hospital. This corresponds with numbers of women requiring multiple procedures found by others ranging from $20 \%$ to $38 \%[13,15,26]$. This number may be overestimated in a tertiary referral center, as the more straightforward cases of mesh exposure that are recovered with a single (office) excision will not be referred.

Recurrence of POP after excision of mesh used in POP surgery was more common with complete excision than with partial excision ( $29 \%$ vs $5 \%)$ and affected the anterior vaginal wall in 7 of $47(15 \%)$ cases of mesh excision from the anterior vaginal wall or vaginal vault. Nevertheless, consistent with a previous study, the majority of patients (86\%) did not have recurrence of POP after mesh excision [13]. Possibly, the formation of scar tissue and fibrosis after mesh insertion surgery provides sufficient support to prevent the recurrence of POP in most patients who had mesh excision, even though in complete mesh excision the interlaced fibrotic tissue may be removed. De novo SUI after mesh excision occurred in $13 \%$ of all patients and reoccurred in 36\% after excision of a suburethral sling, which is consistent with recurrence rates of SUI up to $47 \%$ after suburethral sling excisions in other studies [13, 27]. The small amount of scar tissue around the suburethral tape is often not enough to remain supportive after mesh excision.
In two of our patients (who underwent surgery without the assistance of a urologist), very serious urologic complications occurred. Bilateral ureter lesion occurred in one of the first complete excisions of vaginal mesh performed at our center. This incident has led to a policy in which the urologist is being involved with the more extensive and complex surgeries. Also, the urologist is always involved in the treatment of patients with (suspected) mesh complications affecting the bladder.

Furthermore, intraoperative and postoperative complications (5\% and $16 \%$, respectively) were found relatively frequent in our study compared to the study of MarcusBraun et al. [13]. In their retrospective study of 104 cases of surgical vaginal mesh removal, intraoperative complications occurred only twice (2\%) and postoperative complications in 10 operations $(10 \%)$. However, they did not evaluate patients with mesh excision after sacrocolpopexy. Most complications in our patients occurred in the group of patients who underwent abdominal excision of mesh used in sacrocolpopexy. This is consistent with the findings of South et al. who described that abdominal excision of eroded vaginal mesh after prior abdominal sacrocolpopexy involves more serious complications compared to the vaginal route [26].

The impact of this study is limited by its retrospective design and descriptive nature. It is, therefore, susceptible to recall and interpretation bias. Moreover, since data were collected retrospectively, $32 \%$ of the patients had a followup of only 2 months. Therefore, the postoperative follow-up may have been too short to find recurrences of POP in some cases. Furthermore, $17 \%$ of patients underwent concomitant POP or SUI surgery, which may have reduced the rate of recurrence of POP and de novo SUI we found.

Incidence figures are hard to reproduce in this heterogeneous population, in which patients were often referred by various other hospitals. We were able to estimate the incidence of mesh-related complications only for Prolift ${ }^{\mathrm{TM}}$ inserted at our center. Of the patients included in this study, 20 underwent insertion of Prolift ${ }^{\mathrm{TM}}$ at our hospital between halfway of 2005 and end of 2009. In this period, 180 Prolift $^{\mathrm{TM}}$ meshes were inserted. So, 20 out of 180 (11\%) patients with Prolift ${ }^{\mathrm{TM}}$ inserted at our center developed complications that required excision. All 20 patients initially underwent partial excision, of which 2 required a re-excision (1 partial and 1 complete). As these patients originated from our center, the excisions included both minor and major complications. It can be concluded that, after the insertion of Prolift ${ }^{\mathrm{TM}}, 0.6 \%$ needs excision for severe mesh-related complications and $11 \%$ for minor complications. This incidence number is consistent with numbers found in other studies [9, 13, 21].

Prolift $^{\mathrm{TM}}$ was the most removed mesh material in our patients $(40 \%)$. This could be explained by the fact that, in this region, Prolift ${ }^{\mathrm{TM}}$ is the most used mesh material in 
prolapse repairs. Since it is impossible to generate a denominator for each mesh type and patients referred from other centers, no inference can be made on the overall incidence of mesh complications in the population or the relative complication rates related to individual mesh kits. Different types of mesh may be more likely to erode and others may be easier to excise.

Despite these limitations, this study is strengthened by the relatively large number of patients, the fact that all operations were performed in one center using the same surgical techniques, and the detailed data available from operative reports and medical records. Moreover, this study shows that mesh complications after abdominal sacrocolpopexy and transvaginal mesh present differently and that patients who undergo complete mesh excision have a higher recurrence rate of POP compared to those who required only partial mesh excision, observations that are relatively new to the literature.

\section{Conclusion}

There is a great variety of mesh-related complications, which present differently after abdominal sacrocolpopexy and transvaginal mesh insertion. Excision of mesh to treat these complications is challenging. It often requires extensive dissection and careful operative technique. Relief of symptoms is achieved in the majority of patients.

Although complications do not occur frequently, serious complications may be associated with more extensive or complex surgery. Therefore, we recommend centralization of complete mesh excision, especially when there is involvement of the bladder or rectum. In these cases, the role of the urologist or colorectal surgeon is important to avoid serious organ-specific complications. Checking the ureters after all cases involving any anterior vaginal wall or bladder dissection by cystoscopy to avoid reoperation for ureteric complications should be considered. Partial excision should be preferred in more straightforward cases of mesh exposure with relatively mild symptoms. Complete excision should be reserved for patients with more serious complaints and severe mesh-related complications because of the higher risk of surgical complications and recurrence of POP. The vaginal mesh excision procedure with or without hysteroscopy would be the preferred method to approach patients with symptoms of sacrocolpopexy mesh because it is less invasive and associated with less morbidity than the abdominal approach. Concomitant repair of POP or incontinence surgery should be considered given the rates of recurrence of POP and de novo SUI, especially after complete mesh excision.

The increasing number of inserted meshes for SUI and POP raises concerns. Mesh is successfully used for repair of prolapse, but when complications arise, they may be severe in nature and result in a decrease in quality of life. New meshes are introduced into clinical practice despite the lack of proper studies showing their safety and effectiveness. Moreover, the use of easy-to-do mesh kits lowers the threshold for inexperienced surgeons to start operating with meshes. This can only lead to more complications, which is harmful for the patients. The Food and Drug Administration also has noticed this and has given a firm warning about the use of mesh and how to consult patients [28]. This strengthens us in our opinion that insertion of mesh and the use of kits should be done with utmost skill and only by trained and very experienced surgeons.

The precise impact of mesh-related complications on quality of life has not been researched yet. Therefore, we encourage a future study evaluating the impact of meshrelated complications on the quality of life of patients.

Conflicts of interest None.

Open Access This article is distributed under the terms of the Creative Commons Attribution Noncommercial License which permits any noncommercial use, distribution, and reproduction in any medium, provided the original author(s) and source are credited.

\section{References}

1. Swift S, Woodman P, O'Boyle A et al (2005) Pelvic Organ Support Study (POSST): the distribution, clinical definition, and epidemiologic condition of pelvic organ support defects. Am J Obstet Gynecol 192:795-806

2. Swift SE (2000) The distribution of pelvic organ support in a population of female subjects seen for routine gynecologic health care. Am J Obstet Gynecol 183:277-285

3. Olsen AL, Smith VJ, Bergstrom JO, Colling JC, Clark AL (1997) Epidemiology of surgically managed pelvic organ prolapse and urinary incontinence. Obstet Gynecol 89:501-506

4. Weber AM, Walters MD, Piedmonte MR, Ballard LA (2001) Anterior colporrhaphy: a randomized trial of three surgical techniques. Am J Obstet Gynecol 185:1299-1304

5. Scott NW, McCormack K, Graham P, Go PM, Ross SJ, Grant AM (2002) Open mesh versus non-mesh for repair of femoral and inguinal hernia. Cochrane Database Syst Rev (4):CD002197

6. Nygaard IE, McCreery R, Brubaker L et al (2004) Abdominal sacrocolpopexy: a comprehensive review. Obstet Gynecol 104:805-823

7. Nilsson CG, Falconer C, Rezapour M (2004) Seven-year follow-up of the tension-free vaginal tape procedure for treatment of urinary incontinence. Obstet Gynecol 104:1259-1262

8. Withagen MI, Milani AL, Boon J, Vervest HA, Vierhout ME (2011) Trocar-guided mesh versus conventional vaginal repair in recurrent prolapse; randomized controlled trial. Obstet Gynecol (in press)

9. Nieminen K, Hiltunen R, Takala $T$ et al (2010) Outcomes after anterior vaginal wall repair with mesh: a randomized, controlled trial with a 3 year follow-up. Am J Obstet Gynecol 203:235-238

10. Nguyen JN, Burchette RJ (2008) Outcome after anterior vaginal prolapse repair: a randomized controlled trial. Obstet Gynecol 111:891-898 
11. Sivaslioglu AA, Unlubilgin E, Dolen I (2008) A randomized comparison of polypropylene mesh surgery with site-specific surgery in the treatment of cystocoele. Int Urogynecol J Pelvic Floor Dysfunct 19:467-471

12. Haylen BT, Freeman RM, Swift SE et al (2010) An International Urogynecological Association (IUGA) / International Continence Society (ICS) joint terminology and classification of the complications related directly to the insertion of prostheses (meshes, implants, tapes) \& grafts in female pelvic floor surgery. Int Urogynecol J Pelvic Floor Dysfunct. doi:10.1007/s00192010-1324-9

13. Marcus-Braun N, von Theobald $P$ (2010) Mesh removal following transvaginal mesh placement: a case series of 104 operations. Int Urogynecol J Pelvic Floor Dysfunct 21:423-430

14. Hurtado EA, Appell RA (2009) Management of complications arising from transvaginal mesh kit procedures: a tertiary referral center's experience. Int Urogynecol J Pelvic Floor Dysfunct 20:11-17

15. Margulies RU, Lewicky-Gaupp C, Fenner DE, McGuire EJ, Clemens JQ, Delancey JO (2008) Complications requiring reoperation following vaginal mesh kit procedures for prolapse. Am J Obstet Gynecol 199:678.e1-678.e4

16. Ridgeway B, Walters MD, Paraiso MF et al (2008) Early experience with mesh excision for adverse outcomes after transvaginal mesh placement using prolapse kits. Am J Obstet Gynecol 199:703-707

17. Baessler K, Hewson AD, Tunn R, Schuessler B, Maher CF (2005) Severe mesh complications following intravaginal slingplasty. Obstet Gynecol 106:713-716

18. Maher C, Feiner B, Baessler K, Adams EJ, Hagen S, Glazener CM (2010) Surgical management of pelvic organ prolapse in women. Cochrane Database Syst Rev (4):CD004014
19. Bako A, Dhar R (2009) Review of synthetic mesh-related complications in pelvic floor reconstructive surgery. Int Urogynecol J Pelvic Floor Dysfunct 20:103-111

20. Carey M, Higgs P, Goh J et al (2009) Vaginal repair with mesh versus colporrhaphy for prolapse: a randomised controlled trial. BJOG 116:1380-1386

21. Diwadkar GB, Barber MD, Feiner B, Maher C, Jelovsek JE (2009) Complication and reoperation rates after apical vaginal prolapse surgical repair: a systematic review. Obstet Gynecol 113:367-373

22. Bump RC, Mattiasson A, Bo K et al (1996) The standardization of terminology of female pelvic organ prolapse and pelvic floor dysfunction. Am J Obstet Gynecol 175(1):10-17

23. Kohli N, Walsh PM, Roat TW, Karram MM (1998) Mesh erosion after abdominal sacrocolpopexy. Obstet Gynecol 92:999-1004

24. Mattox TF, Stanford EJ, Varner E (2004) Infected abdominal sacrocolpopexies: diagnosis and treatment. Int Urogynecol J Pelvic Floor Dysfunct 15:319-323

25. Feiner B, Maher C (2010) Vaginal mesh contraction: definition, clinical presentation, and management. Obstet Gynecol 115:325-330

26. South MM, Foster RT, Webster GD, Weidner AC, Amundsen CL (2007) Surgical excision of eroded mesh after prior abdominal sacrocolpopexy. Am J Obstet Gynecol 197:615.e1-615.e5

27. Segal J, Steele AC, Vassallo BJ et al (2006) Various surgical approaches to treat voiding dysfunction following anti-incontinence surgery. Int Urogynecol J Pelvic Floor Dysfunct 17:372-377

28. US Food and Drug Administration (2008) FDA public health notification: serious complications associated with transvaginal placement of surgical mesh in repair of pelvic organ prolapse and stress urinary incontinence. Issued: October 20, 2008. Available at http://www.fda.gov/MedicalDevices/Safety/AlertsandNotices/ PublicHealthNotifications/ucm061976.htm 